\title{
Dynamic Navigation System Design for Networked Electric Vehicles
}

\author{
Frazer McKimm ${ }^{1}$, Manuela Galli ${ }^{2}$, and Veronica Cimolin ${ }^{2}$ \\ ${ }^{1}$ DHS, Shamrock Chambers 1-2 Eustace Street Dublin 2 Ireland \\ ${ }^{2}$ Dipartimento di Bioingegneria, Politecnico di Milano, Milan, Italy
}

\begin{abstract}
Data saturation of satellite navigation systems (already a problem with location based services) will become particularly acute in the emerging area of networked electric vehicles (NEV). Sophisticated energy management and navigation software may solve a technology integration challenge, but it will leave unresolved the usability implications for drivers and fleet operators. These include navigation data specific to commercial electric vehicles; delivery scheduling, routes, times, traffic congestion avoidance, range $\&$ charge levels etc. Many are time dependent factors that complicate interaction with a map based navigation system. They also risk augmenting driver stress and distraction induced errors. This Paper has two objectives. Firstly we examine the problem of information saturation of navigation systems. Secondly we undertook a series of user tests to evaluate an alternative NEV navigation system. The DHS solution is a compressed data feed delivering "just in time" multimodal prompts embedded in the map route. The test results demonstrated improved driver comprehension and reduced driver glance away time from road to navigation system.
\end{abstract}

\section{Research}

Driving is a multi-tasking activity that requires drivers to manage their attention between various driving and non-driving-related tasks. "New studies show that drivers overestimate their own ability to safely multitask, even as they worry about the dangers of others doing it, device makers and auto companies acknowledge the risks of multitasking behind the wheel" [1]. This is especially the case in the use of in-car satellite navigation systems.

More significant is the way map information is presented on a satellite navigation system. These are based upon a 300 year old Ordinance Survey, grid reference maps system. Cartographic representations of an area are even older and more deeply embedded in our subconscious. The designers of these maps assumed the viewer was static (standing or sitting). Driving a vehicle presents quite a different view of reality, one which is dynamic and changing. If one adds dense context based information, then the static map model presents usability problems.

In understanding how we interpret visual information (like Sat Nav maps in a car) we need to understand how the human brain responds to visual stimuli in a dynamic environment. The brain has evolved to interpret a reality generally based upon a near 
static or slowly changing environment. Even in the case of "3D" driver view maps, like the one illustrated below, (Fig. 1) the map is an externalized representation of a reality. It is not how the driver perceives the environment and the navigation "options" as they present themselves.

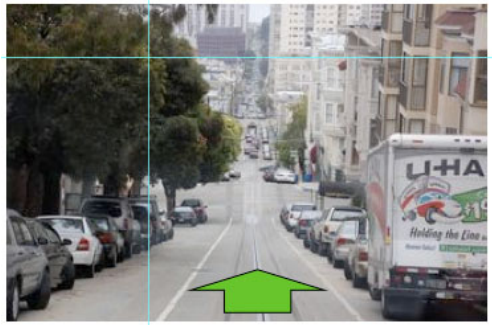

What I see is a dynamic space. The navigator presents me with an externalised abstraction
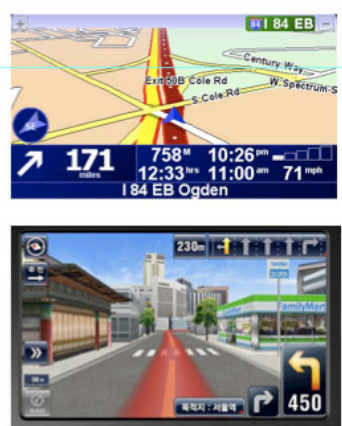

Fig. 1. Route as driver experiences it combines time \& space

For modern men and women, running or rapid movement (outside the context of sport) is a relatively unnatural state of the brain. Decisions and precise calculations are made in this dynamic situation; however in such a state we are fully engaged with our sensory abilities and sensitive to a multitude of surface sounds and external stimuli. From an anthropological or historical perspective the running state was a normal one for primitive man, and the sedentary state of the modern mind is a recent one. Our minds are a summation of a very long evolutionary past and as such, less influenced by recent "modern" history. Carol V. Ward notes; "because the human mind and behavior are products of evolution, we must reconstruct the selective pressures that shaped our lineage in order to understand ourselves today." [2]
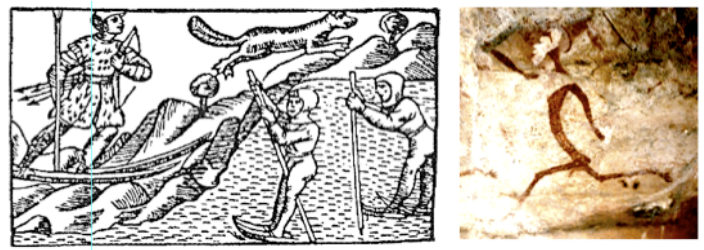

The driving situation as opposed to a normal walking or running one is that from the point of view of the human brain, it's a very abnormal situation.

Fig. 2. Running is part of a normal mind \& body experience

The driving experience in terms of the brain's interpretative abilities can thus be considered an even more abnormal state for us, as our body is static and sensory detection is visually centric. From inside the car, the driver's view or perspective appears as a movement down a "tunnel" like space with a precise time relation.

From an evolutionary perspective, driving is a paradox for the mind. We are rushing towards a target at speed, but our body is static and at the same time senses 
we are moving. There are a number of ways for the driver to view this; we are flying through the air horizontally or we are in a sort of simulated running. This running or rapid movement is to the brain an alert situation, one more related to our primeval "hunter state".

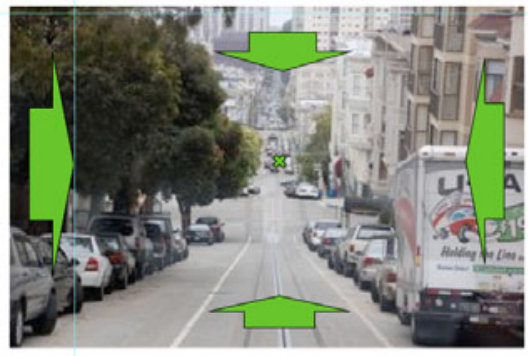

Street tunner in abnormal brian speed fittering situation.

Fig. 3. In driving rapidly through a space anticipation is strong

For the brain, "stress response-also known as the fight-or-flight responseinvolves the release of stress hormones like adrenaline... High levels of adrenaline were meant to serve as emergency fuel generators in times of existential danger, such as when our forefathers were being chased by a wooly mammoth.." [3]

Our theory is that this rapid movement in the car is closer to the "hunter state" (and with its increased adrenaline levels) is the only "similar" condition we have to the driving experience. Drivers of all ages cope with this differently; however, the addition of densely packed data via the navigation system will act to increase apprehension on the part of the driver. Driving along a road forces the mind to make split second adjustments. In this state the body is effectively on alert, the brain is keenly focused on anticipating what is coming towards the driver. Regarding this heightened adrenaline state, much research has been conducted in relation to the "time dilation effect " or the perception of extended time due to heightened levels of adrenaline in the body especially in threatening situations [4]

This offers the interesting postulation that a user now can comprehend and achieve far more in a split second of navigation interaction then in a more relaxed or less adrenaline filled state. We don't agree that a solution to driver distraction caused by data-rich satellite navigation systems lies within this line of reasoning. Research in the area of augmented reality maps where adding "a virtual object with proper inclusions in the real scene" [5] would indicate a viable future solution, but costs are currently too high for mass application.

The visually centric nature of the information in the dynamic car state presents an interpretative dilemma for the brain. Humans have evolved to understand a dynamic running-based state and engage all our senses to interpret the environment in real time in order to make and /or anticipate events affecting our safety. Adrenaline release serves to engender a physical reaction, (flight or fight) one that cannot be done while seated in a car. Secondly, the body's own production of adrenaline is designed for short "abnormal" periods and de facto extended adrenaline periods should not be a standard side effect of driving. Thirdly, we are physically neutered, (seated and still) 
with a truncated sensory capacity, depending primarily on the visual faculty. We are also multi-tasking while in motion: driving, navigating and /or talking on the phone. Whereas the hunter /athlete in us can display great ambidextrous skill while in motion, less can be said of driving and multi-tasking. Driving and talking on the phone, for example, produces a level of cognitive awareness and dexterity analogous with being drunk [6].

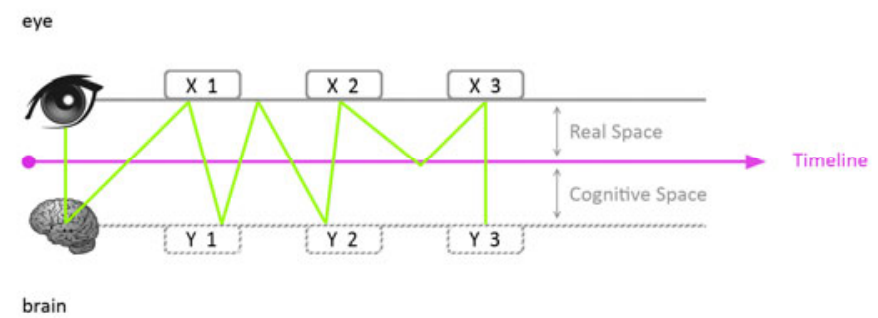

Fig. 4. The brain creates a cognitive version of the time line event sequence

A map view visual model does not take sufficiently into consideration the dynamic, time pressured nature of the car environment. The brain will compare what it is experiencing with what it knows. It has to rely primarily on the visual faculty to "read" both the real and the map representation of events. This does not help the driver in a quasi sensory deprived space to correctly interpret and react to a situation. The subject of sensory deprivation and its various forms has been exhaustively studied since the 1969 John Zubec report " Sensory Deprivation- Fifteen Years of Research". Peter Suedfeld in his introduction noted "perceptual deterioration" as one of many side effects. Though there is a significant difference between the Zubec experiments and a car cabin, parallels exist and relate to the importance of full sensory engagement in reacting to four principal causes of driver distraction and risks of accidents: Visual e.g., focusing on something other than the road, Audible e.g., someone talking. Physical e.g., eating, Cognitive e.g., something that requires you to think about something other than driving. [12] It is reasonable then to state that due to the data rich and dynamic nature of fleet EV navigations systems, driver distraction and incomprehension will increase beyond that of existing navigation systems. This suggests that it is necessary to re-think how an interactive NEV navigation system is designed. Because the driver moves with the car and observes an instantaneous sequence of events, information must be structured and sequenced to match this dynamic. There is (the author believes) a sensory deprivation induced disconnect between an abstracted map model and the lived experience which the brain is normally equipped to deal with. Gallese and Lakoff in their "interactionist theory of meaning" identify the "same neural substrate" or connection between the conceptual and the living experience. "Conceptual knowledge is embodied, that is, it is mapped within our sensory-motor system." [12]. A data feed mechanism is needed to maintain a link between a dynamic but truncated sensory environment and the navigation map. The driver needs context specific, "just in time", multimodal packets of information. As we will discuss later, these "information packets" take the form of landmarks, some iconic or standard, others personal and inserted by the 
driver. Orientation signifiers are central, we believe, to an adaptive and more userinternalized navigation system. As stated earlier, if standard navigation systems are already a usability concern, then Networked Electric Vehicles represent a real challenge.

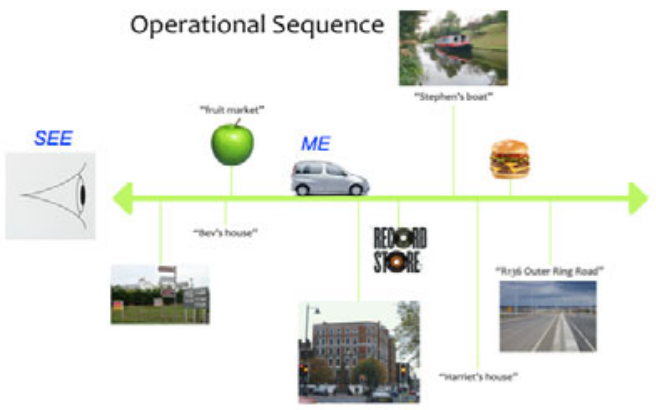

Fig. 5. Above a time line version of event sequence

\section{Networked Electric Vehicles for Fleet use}

A Networked Electric Vehicle is connected to an energy management backend which structures charging of the entire fleet as well as route selection according to electricity grid modulation and energy cost parameters. In the case of standard navigation systems, drivers generally have a single end destination. In the case of fleet NEV's there are multiple destinations and data specific to commercial electric vehicles; delivery scheduling, routes, times, traffic congestion avoidance, range \& charge levels etc. Drivers at the best of times have a limited capacity to manage navigation information in the short time frame of a dynamic environment. This NEV data saturation complicates matters and represents another layer of dynamic information reaching the driver. With electric vehicles, charge point information and range distance will also be factored in.

Observing the above considerations, DHS looked to the sequence and structure of information in its research. The above diagram is a time-line representation of a navigation route where the driver has to reach or pass a number of landmarks. Landmarks have an added value in relation to time management and orientation in a delivery context. They aid a visual and auditory restructuring of the data feed as the characteristics of any in car information presentation should be recognizable, dynamic and compressed. Though a map view of the entire delivery routes would help before driving begins, a compressed "just in time" data presentation would, DHS believes, simplify the navigating experience and reduce driver distraction. In published literature on driver safety there have been numerous studies documenting the effects of distracting tasks while driving. In general, these studies demostrated that distracted drivers have slower responses to critical traffic events and are more likely to miss external events such as changing trafic light, among other effects ([9]; [14]; [7]; [6]) Many studies are also dedicated to the evaluation of the distraction effects related to the use of different kinds of navigation systems. [10]. In particular the results showed that the condition without voice required a higher demand on visual attention. 
The addition of voice substantially reduced the visual attention requirements. These results are reached considering mainly the driver's eye glance behavior in terms of frequency and duration. [11]. Starting from the literature in this topic and from the limits present in current navigation systems, the DHS proposal took into consideration specific time and event stimuli.
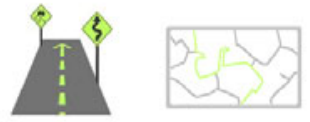

Standard Map
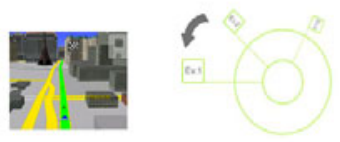

2-ME prototype
$\mathrm{O}=$ multimodal support packets

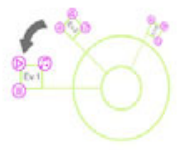

Advanced 2-ME system

Fig. 6. Above 2-ME, early model for just in time information presentation

The aim of the DHS testing work was to evaluate how a dynamic, "just in time", restructured display (termed "2-ME") improves user comprehension, reduce distraction and ultimately increases driver safety. The display uses reconizable "landmarks" which act as prompts where route change decisions need to be taken. These data feeds are supported by auditory prompts located directionally (left, right, straight ahead) to further impove comprenension via multi modal command outputs. [12]

TESTING BACKGROUND: In order to evaluate its "Dynamic Navigation" theory, DHS engaged Dr Galli and Dr Cimolin of the Department of Bioengineering at the Politechnico di Milano, Italy, to design a series of tests which we hoped would prove that this system offered a viable alternative to traditional navigation systems. These tests are covered more comprehensively in a separate paper by the authors "Navigation With Adjusted Multi Modal Data Delivery," whereas the aim here is to explore the application of the concept to Networked Electric Fleet Vehicles.

TESTING METHOD: the drivers followed an urban route in a driving simulator answering to the voice commands given to them by three different navigation systems: the first Satellite navigation traditional system (Sat-Nav), the second (DHS version) based on the use of landmarks able to provide detailed information about the map (2-Me system), and the third (DHS version) with the same landmarks but with a different map representation $(\mathrm{C}-\mathrm{Me})$. The systems were evaluated in terms of the effects on driver distraction. In particular an optoelectronic system for kinematics analysis of head driver movements was used. The number of head movements to the navigator display and the time spent in watching the navigator were key evaluation parameters. In this way researchers were able to identify any distraction occurring in the different navigator modalities.

RESULTS: The 2-Me system is characterized by a low number of head movements to the navigator display and by less time spent by the drivers to read and understand the navigator map presented on it. 
CONCLUSION: The evaluated navigation interface 2-Me shows promising elements for a new navigator interface planning.

APPLICATION: The messages provided by the 2-Me interface are not currently supplied by existing navigation systems. They could possibly be added to them in the near future.

MATERIAL \& METHODS: The effects produced on driving by 3 interface navigation solutions (Sat-Nav, 2-Me interface and C - Me interface) were tested in this work. The Sat-Nav interface is the Satellite navigator system proposed actually by the market (Figure 7). The 2-Me and C-Me interfaces developed by DHS are characterized by the use of specific landmarks to indicate the way that the driver has to follow. Instead of the command proposed by the Sat-Nav system "after $140 \mathrm{~m}$ turn right", in the 2-Me and C-Me modality the command is substituted by "at the church turn right". In this way specific landmarks, related to the landscape, are used in order to better identify the street changes required by the driver. The selection of specific landmarks, related to the urban landscape perceptible as the easiest way by the driver, were chosen in order to answer the compressed information requirements. The two modalities (2-Me and $\mathrm{C}-\mathrm{Me}$ ) differ to each other as described in the following way: 2-Me essentially presents information (to me the driver) in segments that arrives at the driver as he or she drives down a given road (figure 8). C-Me effectively gives the driver an over view (see me - the route) of the trip in one "flash" (figure 9). It connects the memory icons (drawings, photos of places of people) in a continuous strip. We structured our 2-Me and C-Me to allow for the 4-Dimensional Awareness model of the driver being in the car, with data elements arriving in sequence at him/her in a time space "tunnel" situation. We also used pattern recognition and abstraction scale to construct our 2-Me and C-Me solution.

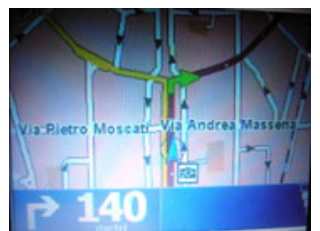

Fig. 7. Sat-Nav interface

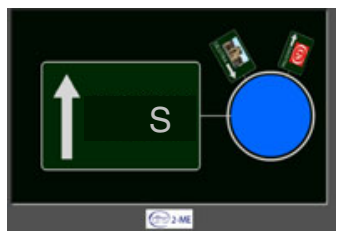

Fig. 8. 2-Me interface

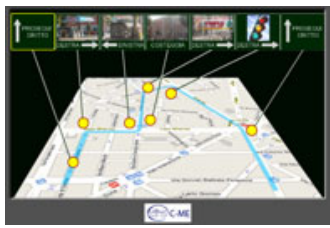

Fig. 9. C-Me interface

20 subjects (11 males; 9 females, mean age $34+/-12$ years) used to a navigation system driving were selected for the tests. The subjects were asked to drive in a simulated car environment, when in front of them a video taken from a real driving experience was presented. In particular the subjects were tested in these tasks:

Task 1- go to the destination following Sat -Nav system.

Task 2- go to the destination following 2-Me interface system.

Task 3- go to the destination following C-Me interface system.

In all the navigation interfaces the given command was both visual and vocal. The aim of this experimental set-up was to evidence how many times and how long the drivers looked at the navigator device in order to better understand the map. The 
subjects were analyzed during the simulated driving, analyzing their biomechanical behaviour using the 3D quantitative kinematic evaluation. In particular the effect produced by the 3 different interface was evaluated analyzing: 1) the number of the head movement corresponding to the number of time in which the driver look to the navigator display 2) the time spent to maintain the position of the head versus the navigation display corresponding to the time spent by the driver looking to the navigator display instead of the road. An optoelectronic system (Elite Bts it) able to measure the $3 \mathrm{D}$ coordinates $(\mathrm{x}, \mathrm{y}, \mathrm{z})$ of passive marker was used for the test.

To know the head movement number to the navigator during the three different tasks , the angle $\alpha$, in the horizontal plane, indentified by the central markers placed on the head and the $\mathrm{x}$ axis (corresponding to the anterior direction), was computed (figure 11). When $\alpha$ is $=0^{\circ}$ it means that the subject is looking to the road in front of him, when $\alpha$ shows a maximum as shown in figure 12 , it means that the subject is looking to the navigator display. This behavior is of particular importance because the more time spent in the evaluation of the screen, the more distraction that is produced by the navigator interface. Starting for the angle $\alpha$ versus time the number and duration of head movements were considered:
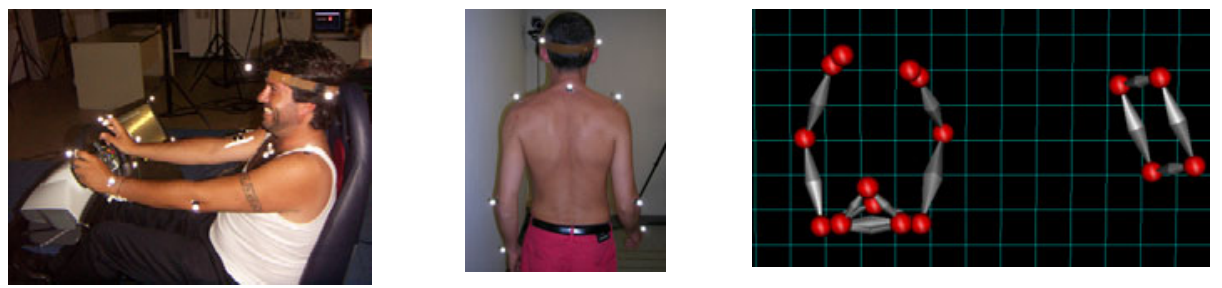

Fig. 10. Marker position (lateral view, back view) and 3D reconstruction (top view during driving)
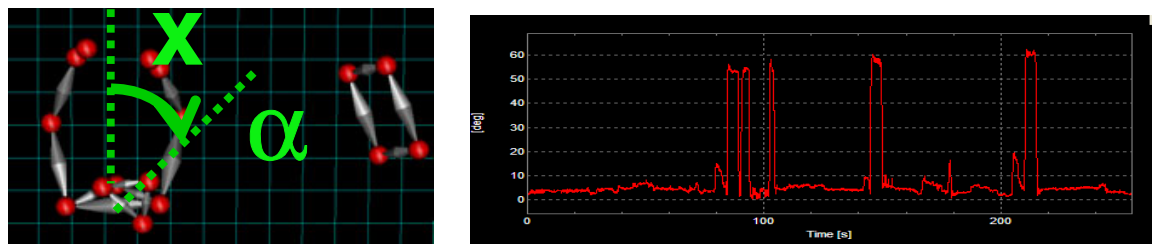

Fig. 11. Identification of angle for the head movement analysis Fig. 12. angles versus time. Peak values correspond to the position of the driver face to the navigator. The plateau duration indicates the time spent by the driver analyzing the navigator screen.

Two kinds of questionnaires were proposed to the drivers. The first one (based on 19 questions) was used to test if the drivers are or not experienced with navigator system driving and with electronic equipments in general. These data are useful in order to establish if the tested subjects could be considered as a homogeneous group. The second one (summarized in Table 1) was proposed in order to know the opinion of the drivers about the use of the three different interfaces. For each question of this questionnaire a score from 1 to 5 (1 not satisfied- 5 fully satisfied) was asked in order to evaluate each kind of Nav system performance. 
Table 1. Proposed questionnaire for the evaluation of the opinion of the drivers in using the three different navigation interfaces (Note All questions are translated from Italian language original)

\begin{tabular}{|l|l|}
\hline 1 & Has it been easy to follow the route? \\
\hline 2 & Were the indications clear? \\
\hline 3 & Were they given early enough? \\
\hline 4 & Were the turning points easy to recognize? \\
\hline 5 & Is the terminology used easy to understand? \\
\hline 6 & Had followed the street instead of the path on the screen? \\
\hline 7 & Was the information enough? \\
\hline
\end{tabular}

\section{Results and Main Considerations}

According to the results of the first questionnaire all the subjects resulted well trained in the use of navigation systems (while driving) and of electronic interfaces (internet, cell phone, ...). It is important in particular to highlight the results of the question $\mathrm{n}$. 6. The drivers recognized that the 2-Me navigator interface allows them to follow the road more and consult the navigation screen less.

Concerning the number of the head movements done and the time spent to look to the navigator system, the 2-Me and C-Me systems produce a lower number of movement towards the navigator, i.e. the trip is better understood in these two modalities with respect to the Sat-Nav. The new interfaces (2-Me and C-Me) also reduced glance away and use of the navigation display information thanks to the introduction of landmarks. The literature shows that any single display glance longer then $2.5 \mathrm{sec}$ is inherently dangerous [16]. The safest of the three tested navigation systems seems to be the 2-Me interface. In this way we confirm that the tests presented in this paper resulted in an improvement of the navigation system interface.

\section{Discussion NEV Application}

Current navigation systems display a density of visual detail that do not match a driver's perception of the dynamic road environment from inside the moving vehicle cabin. This mono sensory input produces a disconnect as the driver tries to interpret an abstracted reality which has already changed in the glance away time from road to display and back again. This was demonstrated in our test results; drivers using standard navigation maps showing an increased propensity to look at the screen. What is already a problem in standard maps risks becoming a serious safety issue with data dense Electric Vehicle navigation systems. The DHS proposal is to restructure the map into a multimodal dynamic data feed which better matches the driver perceived external environment. The main characteristics of the new 2-Me navigation interfaces consists of context based commands. Turn right at the "church" or at a "school" instead of "turn right after 140 meters" or other distance referenced commands. In this way landmarks, (lights, banks or commercial buildings, etc.) quickly identifiable in an urban landscape, in a driving context, are easily recognized by the driver. They are also supported by vocal commands. More 
advanced systems would "internalize" the landmarks by inserting personal references : "turn left at Toms house". DHS would reinforce the multimodality (visual/voice) with tactile or force feed back prompts integrated with this data feed. The mass adoption of Electric Vehicles will bring significant environmental benefits, but also interaction and energy management challenges. This is even more the case with fleet operated Networked Electric Vehicles. These have multiple destinations and time sensitive (energy usage optimized) routes. As a result a careful structuring of navigation data needs to be done to improve usability in relation to safety and also address "range anxiety" concerns. DHS believes that the current "map view" model is an outdated if not misapplied in car solution. We believe the 2-Me concept offers an alternative modality for use in dynamic navigation environments. It is not a finished product and the testing has posed further questions on how the multimodal data feed can be further expanded and more finely calibrated to improve usability. It has also posed questions on how a dynamic and adaptive system can calibrate itself to gender and age variables. These items we wish to address in our next round of testing.

\section{References}

[1] Richtel, M., et al.: NY Times/2009/07/19/technology

[2] Ward, et al.: The Evolution of thought Chapter 18. Body size and intelligence in Hominoid evolution. Department of Anthropology, Department of Pathology and Anatomical Sciences, University of Missouri, Columbia, MO 65211, USA, pp. 335

[3] Bowden, J., et al.: The Most Effective Natural Cures on Earth: The Surprising, Unbiased Truth about What Treatments Work and Why

[4] Wittmann, M., et al.: The neural substrates of subjective time dilation Front. Hum. Neurosci. (2010)

[5] Zenati, N., et al.: ev. Centre of Adv. Technol., Baba Hassen, Algeria (2007) ISBN: 978-14244-1235-8

[6] Strayer, Drews, Crouch, et al.: A comparison of the Cell phone driver and the drunk driver (2006)

[7] Stutts, J.C., Knipling, R.R., Pfefer, R., Neuman, T.R., Slack, K.L., Hardy, K.K.: Guidance for implementation of the AASHTO strategic highway safety plan: A guide for reducing crashes involving drowsy and distracted drivers. Transportation Research Board, Washington, D.C. (2005)

[8] McKnight, A.J., Mcknigh, A.S.: The effect of cellular phones use upon driver attention. Accident Analysis and Prevention 25, 259-965 (1993); Strayer, D.L., Johnston, W.A.: Driven to distraction: dual -task studies of simulated driving and conversing on a cellular phone. Pysic Sci. 12, 462-466 (2001)

[9] Alm, H., Nilsson, L.: Changes in driver behaviour as a function of hands free mobile phones - a simulator study. Accident Analysis and Prevention 26, 441-451 (1994)

[10] Dingus, T.A., Hulse, M.C., McGehee, D.V., Manakkall, N., Fleischmann, R.N.: Driver performance results from the Trav -Tek IVHS camera car evaluation study. In: Proceeding of the Human Factors and Ergonomics Society 38th Annual Meeting, pp. 1118-1122. Human factors and Ergonoimcs society, Santa Monica (1994)

[11] Dingus, T.A., Hulse, M.C., Mollenhauer, M.A., Fleischmann, R.N., McGehee, D.V., Manakkall, N.: Effects of age, system experience, and navigation technique on driving with an advanced traveler information system. Human Factors 39, 177-199 (1997) 
[12] Newell, et al.: Perception 36, 1415-1417 (2007), New insights into multisensory perception

[13] Horrey, W.J., Lesh, M.F., Garabet, A.: Assessing the awareness of performance decrements in distracted drivers. Accident analysis and Prevention 40, 675-682 (2008)

[14] Hancock, P.A., Lesch, M.F., Simmons, L.: The distraction effect of phone use during a crucial driving maneuver. Accident Analysis and Prevention 35, 501-514 (2003)

[15] Lees, M.N., Lee, J.D.: The influence of distraction and driving contex on dirver response to imperfect collision warning systems. Ergonomics 50, 1264-1286 (2007)

[16] Bhise, V.D., Forbess, L.M., Farber, E.I.: Driver behavioral data and considerations in evaluating, Washington D.C (1986)

[17] National Highway Traffic Safety Administration (NHTSA), http: / /www . suvblogger. com/car-safety-guide/are-you-adistracted-driver/ 